\title{
Spectra polarimetry of the motional Stark effect at TEXTOR-94
}

\author{
R. Jaspers, ${ }^{\text {a) }}$ B. S. Q. Elzendoorn, and A. J. H. Donné \\ FOM Instituut voor Plasmafysica "Rijnhuizen," Association EURATOM-FOM, \\ Partner in the Trilateral Euregio Cluster, Postbus 1207, 3430 BE Nieuwegein, The Netherlands \\ T. Soetens \\ Department of Applied Physics, University Ghent, Rozier 44, B-9000 Ghent, Belgium
}

(Presented on 21 June 2000)

The Balmer- $\alpha$ light emitted by neutral particles injected into tokamaks is polarized with respect to the Lorentz electric field experienced by these atoms: $E_{l}=\nu \times B$, known as the motional Stark effect (MSE). On TEXTOR-94 a new MSE system is under development which exploits the full spectral information, along with its polarization. The advantages of exploiting the full spectral information are obvious: beam velocity, observation volume, absolute value of $\mathrm{B}$, the plasma radial electric field and the beam density can in principle all be extracted from the measurement, whereas the polarization can be detected as several lines simultaneously. The TEXTOR-94 MSE system consists of 30 radial channel, of which the full Balmer- $\alpha$ spectrum is measured at two orthogonal angles. This is accomplished by inserting a Glan-Laser prism in the optical path. All the optics is located inside the vessel to improve the radial resolution and minimize changes in polarization due to optical elements. Fibers transfer the light by a vacuum feedthrough towards a spectrometer. It is shown that the measured spectra can be adequately fitted. The accuracy for determining the safety factor $q$ is estimated to be $10 \%-15 \%$ and for the radial electric field to be $30 \mathrm{kV} / \mathrm{m}$. (C) 2001 American Institute of Physics. [DOI: 10.1063/1.1319611]

\section{INTRODUCTION}

Over the past few years the tokamak research has seen exciting advances in the improvement and understanding of confinement. Internal transport barriers in either the ion ${ }^{1}$ or electron ${ }^{2}$ channel have been observed, which most likely occur near rational values of the safety factor $q$. Optimizing the magnetic shear profile and thus the $q$-profile yielded enhanced performance. ${ }^{3}$ The reduction of transport in the $\mathrm{H}$ (Ref. 4) and $\mathrm{VH}^{5}$ modes shows to be correlated with the shear in the radial electric field acting to suppress turbulence. These observations ask for an accurate measurement of the $q$ profile and the radial electric field to deduce the exact mechanisms of confinement improvement.

Diagnostics based on the motional Stark effect have been demonstrated to allow measurements of the $q$ profile ${ }^{6-8}$ with sufficient accuracy and recently even the radial electric field was revealed in a more advanced setup. ${ }^{9}$ This diagnostic is based on the Balmer- $\alpha$ light emitted by injected highenergy neutral hydrogen particles. The Lorentz electric field $\mathbf{E}_{1}=\boldsymbol{\nu} \times \mathbf{B}(\nu$ being the particle's velocity and $B$ the magnetic field) experienced by these particles leads to a splitting of the emission in polarized lines. Measuring these quantities reveals the electric field vector $\mathbf{E}$ consisting of $\mathbf{E}_{\mathbf{l}}$ (from which B can be constructed) and possibly $\mathbf{E}_{\mathbf{r}}$.

All presently operational MSE diagnostics use a narrow band interference filter to select one of the Stark lines and determined the polarization by using two crossed photoelastic modulators (PEMs). Although this modulation technique leads to a very precise measurement of the polarization

${ }^{a)}$ Electronic mail: r.jaspers@fz-juelich.de angle, it has two major drawbacks: (1) small (5\%) changes in beam velocity would shift the Stark lines out of the filter wavelength and (2) all other spectral information (splitting, polarization of other components, etc.) is lost. Therefore at TEXTOR- $94^{10}$ another approach is chosen which determines the polarization of the full spectrum. The realization of this concept is discussed in this paper.

This article is organized as follows. In Sec. II a brief review of the characteristics of the Balmer- $\alpha$ spectrum is given. Then the TEXTOR-94 MSE diagnostic is introduced and the technical details of the components are described. The first results are presented in Sec. IV where typical spectra are shown and analyzed. Finally in Sec. V the advantages and drawbacks of this method of spectra polarimetry are discussed.

\section{BALMER- $\alpha$ SPECTRUM}

A typical Balmer- $\alpha$ spectrum in the presence of the beam emission is shown in Fig. 1. The main features of this spectrum of the $n=3 \rightarrow n=2$ transition are the cold $(\sim 1 \mathrm{eV})$ emission line from the neutral $\mathrm{H}$ and $\mathrm{D}$ atoms from the plasma edge at $\lambda_{h}=656.28 \mathrm{~nm}$ and $\lambda_{d}=656.11 \mathrm{~nm}$. This spectrum is broadened by a passive charge exchange contribution, but in case a neutral beam is injected, the main broadening is due to the active charge exchange contribution. From that part, a local measurement of the hydrogen/ deuterium temperature and rotation is possible. ${ }^{11}$ Doppler shifted from this plasma emission is the beam emission, originating from impact excitation between beam neutral and plasma electrons and ions. This emission consists of three groups with different Doppler shifts, corresponding to neu- 


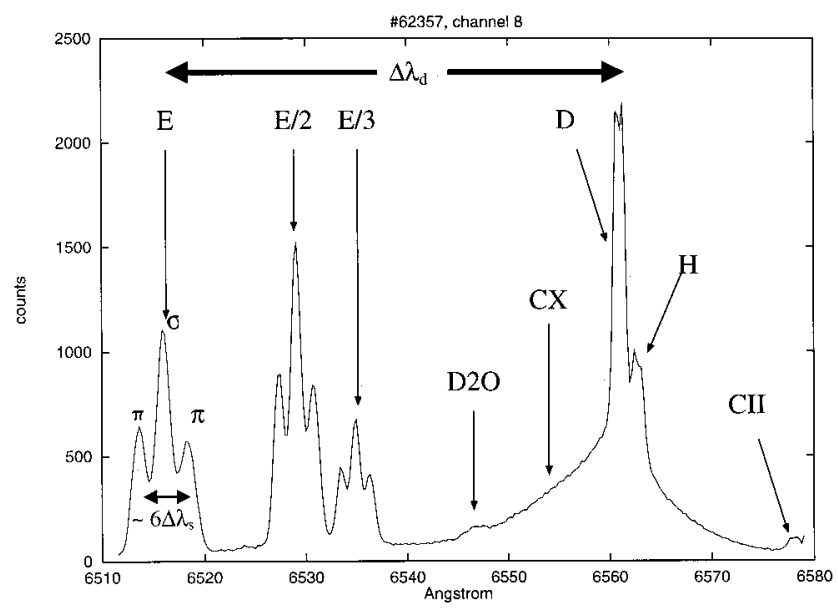

FIG. 1. Typical Balmer- $\alpha$ spectrum measured on TEXTOR-94. The spectrum consisted of a cold emission from the plasma edge of hydrogen and deuterium emission, a passive and active charge exchange component, and three groups of beam emission is Doppler shifted from the edge emission. Moreover, this emission is splitted due to the motional Stark effect. The beam emission is polarized.

trals at the full, half, and third acceleration energy (due to the presence of $\mathrm{H}^{+}, \mathrm{H}_{2}^{+}$, and $\mathrm{H}_{3}^{+}$in the ion source). Each group is split in 15 distinct lines due to the electric field experienced by the neutrals. Nine of them have an observable intensity and their relative strength has been calculated. ${ }^{12}$ The central three of these lines are $\sigma$-polarized (polarized $\perp E$, corresponding to the transition $\Delta m= \pm 1, m$ being the quantum number) and the other $\pi$ polarized (polarized parallel to $E$, corresponding to the transition $\Delta m=0$ ). All these lines are separated by an amount $\Delta \lambda_{s}$ due to the electric field $\mathbf{E}_{l}$ $=\boldsymbol{\nu} \times \mathbf{B}=\boldsymbol{\nu}_{\text {beam }} \mathbf{B}_{\perp}$ (assuming no $E_{r}$ present), which in case of injection in the equatorial plane under an angle $\Omega$ with the torodial direction is given by

$$
\Delta \lambda_{s}=\frac{3 a_{0} e \lambda_{0}^{2} \nu_{\text {beam }}}{2 h c} \sqrt{B_{T}^{2} \sin ^{2} \Omega+B_{p}^{2}},
$$

where $a_{0}$ is the Bohr radius, $e$ the electron charge, $\lambda_{0}$ the unshifted Balmer- $\alpha$ line, $\nu_{\text {beam }}$ neutral beam velocity, $h$ Planck's constant, $c$ velocity of light, $B_{t}$ toroidal magnetic field, and $B_{p}$ poloidal magnetic field.

Although the total beam emission thus consists of 27 Gaussians, the number of fit parameters can be drastically reduced. A total of nine free parameters has and can be determined:

(i) The angle $\beta$ between line of sight and neutral beam is determined from the position of the central $\sigma_{0}$ line of either of the three energy components. (Note that the additional information present in the positions of the $\sigma_{0}$ line of the other two components can be used to determine an exact wavelength scale even when the $\lambda_{0}$ line is not recorded). Apart from the fact that this angle $\beta$ will be used to convert the measured polarization angle $\gamma_{s}$ to the magnetic pitch angle $\gamma$, it can be utilized as well to have an accurate in situ determination of the observation volume. Knowing the location of the observing optics and the neutral beam path, the major radius $R$ of the observation volume depends only on $\beta$. (ii) The Stark splitting $\Delta \lambda_{s}$. This parameter directly reveals $B_{\perp}$.

(iii) The width of the individual lines is determined by the instrumental width of the spectrometer. Divergence effects of the neutral beam will have an additional broadening which scales with the velocity. For the description of the TEXTOR-94 spectra this leads only to a minor improvement of the fit and was not always applied in the analysis.

(iv) For each energy component the intensity of the $\pi$ and $\sigma$ components have been treated as two independent quantities resulting in a total of six free parameters. The relative ratios between each group of $\pi$ or $\sigma$ components have been calculated following the treatment of Ref. 12. If no polarizing element is present in the optical path, comparison of these components could reveal the direction of the electric field, since the angle $\theta$ between the electric field vector and the viewing line is given by

$$
\tan \theta= \pm \sqrt{\frac{2 I_{\pi}}{I_{\sigma}-I_{\pi}}} .
$$

In this way it might be possible to have only this angle $\theta$ as a free parameter and reduce the number of free parameters for the intensities to 4 . This however is not done, which makes it possible to observe the variation in the angle $\theta$ as determined by the different energy components and in case large radial electric fields $E_{r}$ would be present, the angle $\theta$ will be a function of the beam velocity and cannot be taken constant. Apart from the angle $\theta$, the ratio of intensities of the different energy components can be used to calculate the beam composition in the plasma. Absolute values might even be used to calculate beam densities or power deposition profiles. This is for the TEXTOR-94 case successfully demonstrated in Ref. 13. This subject, however, will not be treated in the remainder of the article.

\section{TEXTOR-94 SETUP}

To determine the direction of the Lorentz field application of Eq. (2) will be sufficient. However, this method is not expected to be very reliable since (a) some optical elements will have different transmission or reflection for $\sigma$ or $\pi$ radiation, through which the measured angle $\theta$ will deviate from the actual $\theta$ and (b) Eq. (2) assumes that all lines will be statistically populated, whose condition might not be fulfilled at low plasma density operation. A more accurate way of measuring the direction of $E_{l}$ would be to measure the polarization of the individual lines, which is an immediate representation of the direction of $E_{l}$.

A complete determination of the polarization would require obtaining the four Stokes parameters. These can be constructed from, for instance, the total intensity, two linear polarizations, and a circular polarization and determines the full state of the light vector. A diagnostic based on complete Stokes polarimetry would yield all information necessary to account for changes of the polarization due to optical elements and is thus self-calibrating as shown by Voslamber. ${ }^{14}$

Based on these ideas a proof-of-principle experiment was performed on TEXTOR-94, which consisted of four radial channels, each measuring the full spectrum at four dif- 


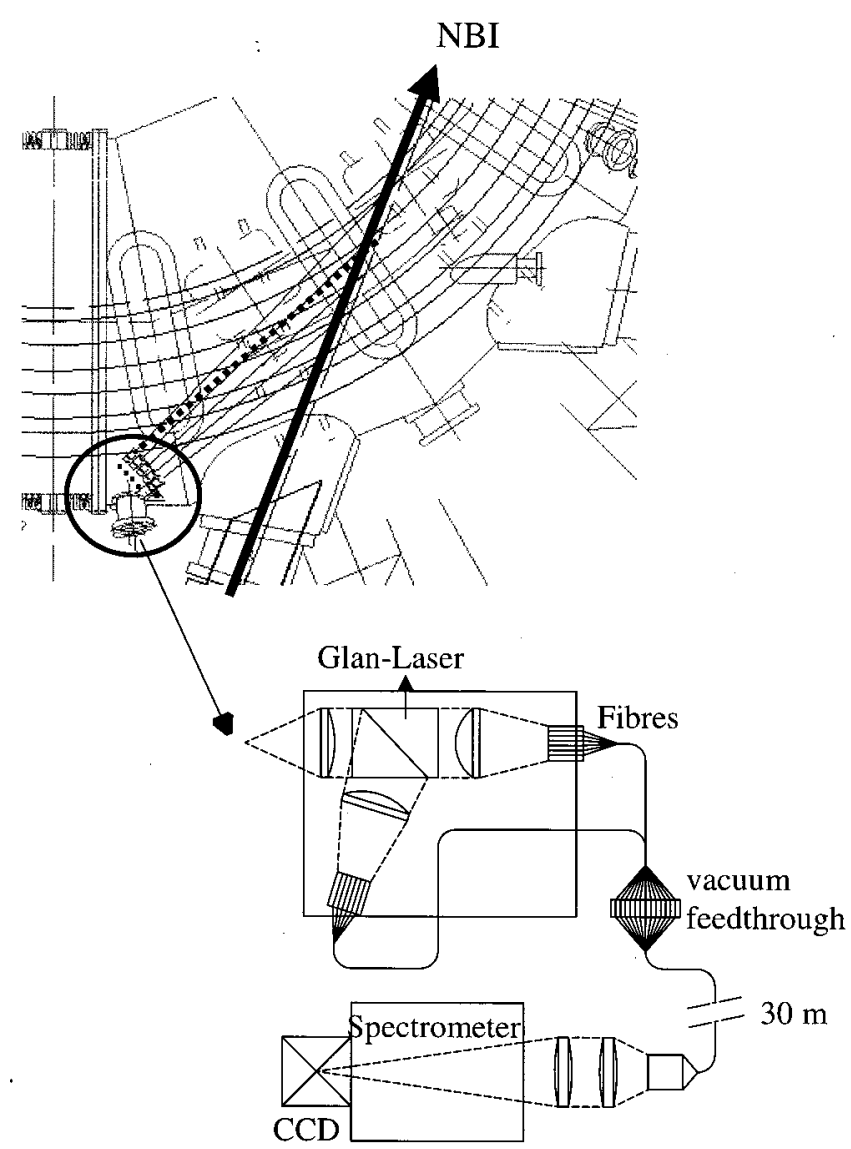

FIG. 2. Sketch of the setup of the TEXTOR-94 MSE system. The neutral beam is viewed from behind, yielding a good radial resolution over a large region. The optics are divided over five identical modules, all equipped with two sets of six fibers. The Glan-Laser beam splitter acts as polarizer. The fibers are imaged onto a spectrometer and the CCD camera records 24 spectra simultaneously in $50 \mathrm{~ms}$.

ferent polarizations. ${ }^{15}$ Although the method in principle worked, the accuracy was insufficient to determine precise values for the safety factor. The two main reasons where the rather low measured intensity as a result of the beamsplitters used and even more important the very limited spatial resolution.

The new setup is therefore optimized for good radial resolution. Since for the optimal position no window was available it was decided to measure the polarization inside the vacuum vessel and transfer the light out by fiber optics. Since in this setup no mirror is present it is sufficient to measure the polarization under only two different angles. These considerations lead to the following concept, as seen in Fig. 2. A total of 30 radial channels (divided over five modules) cover the plasma from the center to the edge, with a radial variation of less than 5\% over the full profile. This is accomplished by inserting all optical components inside the vessel looking towards the beam at different angles for different channels. The polarization is obtained by a polarizing beam splitter of the type Glan-Laser prisms $(20 \times 20 \times 27$ $\mathrm{mm} a$-BBO crystal from CASIX) in the optical path. They were the only alternative to operate at the high temperatures of the TEXTOR liner $(T=650 \mathrm{~K})$. The polarization charac- teristics of this crystal require a collimated beam of less than $6^{\circ}$. The light is focused into 60 quartz/quartz fibers with polyimide coating and a core/cladding diameter of 600/660 $\mu \mathrm{m}$, and numerical aperture $=0.23$. A special fiber feedthrough is constructed consisting of two metal plates having 60 holes drilled somewhat larger than the fiber diameter. Between these plates is silicone rubber to achieve the required vacuum conditions, when the plates are pressed together. Outside the tokamak the fibers can all be individually connected to two sets of fibers transferring the light to either a Littrow spectrometer or a linear array. The use of the spectrometer $(f=0.75 \mathrm{~m}, F /$ No. $=4.5)$ equipped with a $2 \mathrm{D}-\mathrm{CCD}$ camera limits the time resolution to $50 \mathrm{~ms}$ (for recording 24 spectra). For that reason a provision is made to couple the optical fibers to a narrow band interference filter followed by a linear array. This increases the time resolution to less than $1 \mathrm{~ms}$, at the cost of loosing the spectral information.

\section{RESULTS}

To illustrate the working of the MSE diagnostic three spectra, all measured simultaneously at the same location, are shown in Fig. 3. One spectrum records the polarization under $0^{\circ}$, the second under $90^{\circ}$ and finally one gives the total spectrum (one module was not equipped with a Glan-Laser prism). These three channels have been selected since they are in the vicinity of the sawtooth inversion radius and a $q$ value of about 1 is anticipated.

For the circular cross section of the plasma at TEXTOR-94 the magnetic field direction $\gamma$ is directly related to $q$ by: $\tan \gamma=r /(R q)$. Three different ways to obtain this $q$ have already been addressed in Sec. II and are (in order of accuracy of the measurement):

(a) the ratio of one of the $\sigma$ intensities measured at the two angles $0^{\circ}$ and $90^{\circ}$ :

$$
\tan \gamma_{p}=\frac{I\left(90^{\circ}\right)}{I\left(0^{\circ}\right)}=\tan \gamma \frac{\cos (\Omega-\alpha)}{\sin \Omega},
$$

where $\alpha, \Omega, \gamma$, and $\gamma_{p}$ ar the angle between the line of sight and the toroidal direction, the angle between the neutral beam and the toroidal direction, the magnetic pitch angle and the measured polarization angle, respectively. Note, that since $\Omega \approx 30^{\circ}$ and $\alpha \approx 25^{\circ}$, the measured angle is about a factor of 2.0 larger than the magnetic pitch angle. This resulted for the example shown in $q=1.1 \pm 0.2$ if the $\sigma$ lines were analyzed. Within the error bar no systematic difference between the measured polarization angle of the full, half, and third energy components could be seen, indicating the $E_{r}$ to be small at this position.

(b) Comparing the intensity from the $\pi$ and $\sigma$ components for the spectrum measured directly without the polarizing splitter, using Eq. (2). This yielded $q=0.6 \pm 0.3$.

(c) Directly from Stark splitting $\Delta \lambda_{s}$, assuming the toroidal magnetic field is known, by applying Eq. (1) gives $q=0.5 \pm 0.4$.

The accuracy shows that only method a can be used to obtain quantitative results on $q$. Although the Stark splitting $\Delta \lambda_{s}$ can be determined with an accuracy of less than $1 \%$, the 

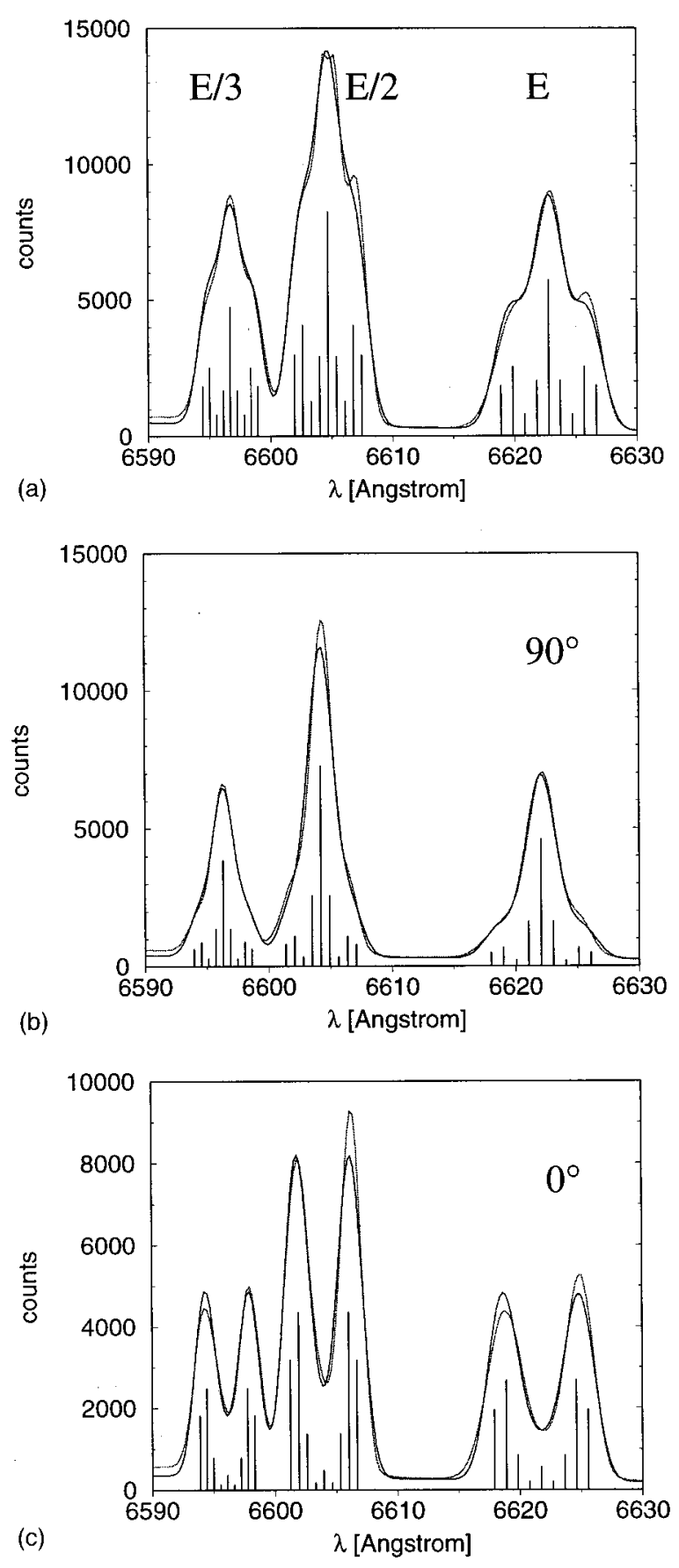

FIG. 3. Three spectra of the beam emission measured at the same time at the same plasma radius. (a) Complete spectrum, no polarizer inserted in the optics; (b) polarized under $90^{\circ}$; and (c) polarized under $0^{\circ}$. The measured spectra and the fit results are overlaid. Good agreement is observed. The intensities of the individual lines are indicated as well. From the intensity ratio of the latter two the magnetic pitch angle is deduced to be $q=1.1 \pm 0.2$.

uncertainty in $B_{t} \sin \Omega$ is a bigger effect than the total $B_{p}$ contribution itself and method $\mathrm{c}$ is thus only used calculating $B_{t} \sin \Omega$.

To reduce systematic errors in the measurements an experiment was performed by injecting the neutral beam in the gas filled tokamak, with only the toroidal magnetic field switched on. This yields an offset angle for the polarization measurements. Moreover, this can also be used to determine the Stark splitting in the case of an absence of poloidal magnetic field. Then a measured value of $B_{t} \sin \Omega$ is obtained. However, since this was done at a different magnetic field than shown here, this has not yet been included in the analysis.

\section{DISCUSSION}

The advantages of exploiting the full spectral information compared to the interference filter based PEM systems are obvious:

(i) the system is insensitive to variations in beam velocity or magnetic field;

(ii) the Doppler shift is measured as well, which allows to determine the observation volume directly from the spectrum;

(iii) the polarization is measured at several lines simultaneously, increasing the accuracy of the deduced direction of the magnetic field;

(iv) the Stark splitting is obtained allowing the computation of the magnitude of the magnetic field;

(v) if in addition to $E_{l}$ a radial electric field is present in the plasma this can, albeit with limited accuracy, be determined by comparing the polarization direction of two different energy components of the neutral beam. (Note here that although the Stark splitting is mainly determined by the strong $\nu_{\text {beam }} B_{t} \sin \Omega$ term, the $E_{r}$ term adds to the $\nu_{\text {beam }} B_{P}$ term and therefore the effect on the polarization of the $E_{r}$ term is of similar importance as the $B_{P}$ term.) During normal plasma operation the radial electric field is expected to be related to the toroidal velocity $v_{t}$ which amounts to about $100-150 \mathrm{~km} / \mathrm{s}$. In the case of a deuterium neutral beam than the importance of the $E_{r}$ term is given by the ratio $v_{t} / v_{\text {beam }} \approx 0.05-0.1$. Nevertheless during transition to improved confinement modes or in the presence of strong gradient associated with transport barriers larger electric fields might be expected and will be noticeable on the MSE measurements.

When the full four Stokes parameters are determined, the system is self-calibrating with respect to polarization changes due to optical element, as discussed by Voslamber. ${ }^{14}$ This option however is not presently used at TEXTOR-94.

The drawbacks of the method of spectra polarimetry are found in (i) the lower time resolution possible; (ii) the use of spectrometers reduces the effective throughput compared with the interference filter method (but this is partly compensated for by the use of more spectral information); and (iii) a more complicated analysis is necessary which prohibits having an output signal directly proportional to the pitch angle.

Also, for the $E_{r}$ determination better-suited diagnostics are available such as the heavy ion beam probe, ${ }^{16}$ but this leads to complicated and expensive systems whereas here this information is obtained automatically.

In conclusion, it is believed that only by an accurate assessment of the full Balmer- $\alpha$ spectrum an unambiguous determination on the Lorentz electric field can be made and this has motivated the choice of the present setup. 
${ }^{1}$ C. M. Greenfield et al., Phys. Plasmas 4, 1596 (1997).

${ }^{2}$ M. R. de Baar, G. M. D. Hogeweij, N. J. Lopes Cardozo, A. A. M. Oomens, and F. C. Schüller, Phys. Rev. Lett. 78, 4573 (1997); N. J. Lopes

Cardozo et al., Plasma Phys. Controlled Fusion 39, B303 (1997).

${ }^{3}$ C. Gormezano, Plasma Phys. Controlled Fusion 41, B367 (1999).

${ }^{4}$ F. Wagner et al., Phys. Rev. Lett. 49, 1408 (1982).

${ }^{5}$ K. H. Burrell, M. E. Austin, C. M. Greenfield, L. L. Lao, B. W. Rice, G. M. Staebler, and B. W. Stallard, Plasma Phys. Controlled Fusion 40, 1585 (1998).

${ }^{6}$ F. M. Levington, Rev. Sci. Instrum. 70, 811 (1999).

${ }^{7}$ D. Wroblewski and L. L. Lao, Rev. Sci. Instrum. 63, 5140 (1992).

${ }^{8}$ N. C. Hawkes, K. Blackler, B. Viaccoz, C. H. Wilson, J. B. Migozzi, and B. C. Stratton, Rev. Sci. Instrum. 70, 894 (1999).

${ }^{9}$ B. Rice, D. G. Nilson, K. H. Burrell, and L. L. Lao, Rev. Sci. Instrum. 70,
815 (1999).

${ }^{10}$ H. Soltwisch et al., Plasma Phys. Controlled Fusion 26, 23 (1984).

${ }^{11}$ E. Busche, H. Euringer, and R. Jaspers, Plasma Phys. Controlled Fusion 39, 1327 (1997).

${ }^{12}$ W. Mandl, R. C. Wolf, M. G. von Hellermann, and H. P. Summers, Plasma Phys. Controlled Fusion 35, 1373 (1993).

${ }^{13}$ T. Soetens, R. Jaspers, M. Von Hellermann, G. Van Oost, and E. Desoppere, 27th EPS Conference on Controlled Fusion and Plasma Phys., Budapest, 2000, pp. 4-108.

${ }^{14}$ D. Voslamber, Rev. Sci. Instrum. 66, 2892 (1995).

${ }^{15}$ T. Soetens, R. Jaspers, and E. Desoppere, Rev. Sci. Instrum. 70, 890 (1999).

${ }^{16}$ I. S. Bondarenko et al., Rev. Sci. Instrum. 68, 312 (1997). 